\section{AB1092 SATISFACTION AND BELIEF REGARDING TREATMENT WITH INTERSTITIAL LUNG DISEASE AND RHEUMATIC DISEASES IN MULTIDISCIPLINARY OUTPATIENT CLINIC}

J. Jung ${ }^{1}$, S. Jung ${ }^{1}$, S. Kim ${ }^{2}$, J.R. Choi ${ }^{3}$, H.-S. Kim ${ }^{1} .{ }^{1}$ Internal medicine, the Soonchunhyang University Seoul Hospital, Seoul; ${ }^{2}$ Internal medicine, Gangneung Asan Medical Center, Gangneung; ${ }^{3}$ Internal medicine, Pohang Saint Mary's Hospital, Pohang, Korea, Republic Of

Background: Among rheumatic disease-related lung disease, interstitial lung disease (ILD) is the most prevalent and contributing to the mortality and morbidity. Increasing number of recent reports dealing with ILD of rheumatoid arthritis (RA), Sjögren's syndrome (pSS) and systemic sclerosis (SSc), multidisciplinary discussions (MDDs) have been growing interest for diagnostic accuracy with dedicated service. There was little evidence indicating that multidisciplinary outpatient clinic resulted in improvements in clinical outcomes including satisfaction and belief.

Objectives: We have designed validated a scale of evaluating coping strategies about satisfaction and belief in patient of ILD and rheumatic disease with multidisciplinary approach.

Methods: From December 2015 to September 2016, we evaluated 20 patients of rheumatoid disease with ILD and 20 patients of idiopathic pulmonary fibrosis. Patient perceptions of illness, treatment beliefs, and moods were measured via the multiple choice questionnaires presenting brief Illness perception questionnaire, beliefs about medicines questionnaire, and patient health questionnaire 2 for comparing the effectiveness of MDDs and routine ILD management.

Results: In univariate analysis, beliefs in necessity and concerns of medication differed significantly high in multidisciplinary outpatient clinic for people with ILD (intentional or unintentional). When controlling for other factors that may impact medication nonadherence, more belief in necessity of medication and greater positive emotional response to disease were presented in multidisciplinary outpatient clinic for people with ILD (OR 1.51, Cl 1.01-1.82).

Conclusions: The MDD including rheumatologist and pulmonologist allows a satisfactory management comparing routine ILD management. It showed better coping improvements about the emotional distress, pain, and beliefs about treatment. Further research to investigate long-term clinical outcomes of multidisciplinary outpatient clinic for people with ILD is required, overlying the enhancement of mutual communication.

\section{References:}

[1] Vliet Vlieland TP. Multidisciplinary team care and outcomes in rheumatoid arthritis. Curr Opin Rheumatol 2004;16(2):153-6.

[2] Vliet Vlieland TP, Hazes JM. Efficacy of multidisciplinary team care programs in rheumatoid arthritis. Semin Arthritis Rheum 1997:27(2):110-22.

[3] Oberai B, Kirwan JR. Psychological factors in patients with chronic rheumatoid arthritis. Ann Rheum Dis 1988;47(12):969-71.

[4] Cramer JA, Roy A, Burrell A, et al. Medication compliance and persistence: terminology and definitions. Value Health 2008;11(1):44-7 doi: 10.1111/j.1524-4733.2007.00213.x [published Online First: Epub Date]|.

[5] Pakas I, loannidis JP, Malagari K, et al. Cyclophosphamide with low or high dose prednisolone for systemic sclerosis lung disease. J Rheumatol 2002;29(2):298-3046.

[6] Mouthon L, Berezne A, Guillevin L, et al. Therapeutic options for systemic sclerosis related interstitial lung diseases. Respir Med 2010;104 Suppl 1:S5969 doi: 10.1016/j.rmed.2010.03.018 [published Online First: Epub Date]|.

Disclosure of Interest: None declared

DOI: 10.1136/annrheumdis-2017-eular.4179

\section{AB1093 BIOSIMILAR INFLIXIMAB IN RHEUMATOLOGY PRACTICE - THE} CYPRUS EXPERIENCE

J. Joseph, M. Solonos, M. Michaelides, V. Hadjiroussos, V. Skoutellas,

N. Zannettou, C. Christodoulou, E. Mina, A. Therapontos, D. Nikiforou,

S. Psarellis, S. Symeonidou, A. Tsirogianni, M. Michaelidou. Cyprus

Rheumatology Association, Nicosia, Cyprus

Background: Infliximab ('Remicade') has been authorized in the EU since 1999. It has a license for use in major rheumatic diseases and inflammatory bowel disease. The biosimilar 'Inflectra' received its license in Europe in September 2013. It was first launched in Central and Eastern Europe, and some smaller Western European markets due to earlier patent expiry. Inflectra was introduced to Cyprus in 2014 and soon after that, every patient with rheumatoid arthritis, ankylosing spondylitis and psoriatic arthritis would receive this agent when a biologic agent was required. At first there was significant concern amongst the medical community regarding efficacy and safety of biosimilars. Careful observation and pharmacovigilance are therefore required to analyse the effectiveness and safety of biosimilar agents since the significant price difference means that they will be used extensively from now on.

Objectives: The aim of this study was to record statistics of use, effectiveness and safety of the first biosimilar 'Inflectra' following its introduction in Cyprus. Methods: Cypriot rheumatologists completed an online form for every patient who was prescribed 'Inflectra' from the introduction of the biosimilar in 2014, until late 2016. Collected data included patient characteristics, diagnosis, whether the patient remains on the drug or not, reasons for discontinuation, side effects and physician impression of effectiveness.

Results: 160 patients were entered. Male: Female ratio was roughly equal and
$90 \%$ were taking a biologic for the first time ('biologic naive'). Indications were Rheumatoid arthritis in $40 \%$, Ankylosing spondylitis in $33 \%$, psoriatic arthritis and other spondyloarthropathies in $20 \%$ and a few patients were treated for eye disease and other 'off-label' indications. At the time of recording, $25 \%$ of patients were on the drug for less than 3 months, 25\% 3-6 months, 25\% 6-12 months and $25 \%$ over a year. Overall $30 \%$ of patients had to discontinue and $70 \%$ remain on the drug. Of those who discontinued, $65 \%$ did so within 6 months of starting. Of all patients treated with Inflectra, 10\% stopped due to side effects, $7 \%$ had immediate non-effectiveness and 3\% had secondary lack of efficacy. $80 \%$ of patients experienced no adverse effect. Amongst the 160 patients, recorded adverse events included 8 infections, 8 skin rashes, 9 headaches and 5 severe allergic reactions. Amongst the infections were 2 respiratory, 2 herpes zoster, 1 sinusitis 1 cellulitis, 1 urinary and 1 gastroenteritis. 94\% knew they were taking a biosimilar and $80 \%$ had no objection. Patient concerns included safety and effectiveness. The treating doctor was 'quite' or 'very' happy with achievement of the therapeutic target in $66 \%$ of cases and 'unhappy' in only $15 \%$.

Conclusions: Despite understandable concerns with the introduction of biosimilars, the experience with our first 160 patients was good; numbers remaining on the drug and adverse effects were similar to previous large studies of infliximab. Rheumatologists feel happy the therapeutic target has been met in a significant majority of cases. Continuous observation and pharmacovigilance are required as with the introduction of any new agent.

Disclosure of Interest: None declared

DOI: 10.1136/annrheumdis-2017-eular.4200

\section{AB1094 ASSOCIATION BETWEEN USE OF TRADITIONAL CHINESE MEDICINE AND MEDICATION ADHERENCE AMONG CHINESE-AMERICAN RHEUMATOLOGY PATIENTS}

K. Sun ${ }^{1}$, H. Tian ${ }^{2}$, Y.J. Chang ${ }^{3}$, L. Lee ${ }^{1}$, J.C. Leng ${ }^{4}$, L.A. Mandl ${ }^{1} .{ }^{1}$ Hospital for Special Surgery; ${ }^{2}$ New York University School of Medicine; ${ }^{3}$ Mount Sinai Beth Israel; ${ }^{4}$ Memorial Sloan Kettering Cancer Center, New York, United States

Background: Chinese-Americans (CA) are a fast-growing US immigrant group with high utilization of Traditional Chinese Medicine (TCM) and worse SLE and RA outcomes than Caucasians $(1,2)$. The effect of TCM use on adherence to prescribed western medications for systemic rheumatic diseases is unknown. Objectives: To evaluate whether TCM use is associated with adherence to western medicines prescribed for systemic rheumatic diseases among CA patients. Methods: Patients with systemic rheumatic diseases were recruited from 2 rheumatology clinics that serve a predominantly CA immigrant population. Inclusion criteria were speaking Mandarin or English and having medication(s) prescribed by the rheumatologist. TCM use, adherence, Patient-Reported Outcomes Measurement Information System (PROMIS) domains, and other variables were assessed using validated instruments available in English and Chinese. Adherence was classified as high or medium/low based on the 8-item Morisky Medication Adherence Scale (3). Medication complexity was assessed using the Medication Regimen Complexity Index (MRCl) (4); higher score indicates more complexity.

Results: 177 enrolled, mean age 54 (range 20-97), 62\% female, 73\% <high school education, $75 \%$ Medicaid (subsidized insurance), and only $18 \%$ spoke English. Diagnoses were RA (43\%), SLE (17\%), SpA (16\%), Sjogren's $(8 \%)$, gout/CPPD (6\%), and other (10\%). 49\% reported TCM use in the past year, most commonly tuina massage (48\%), acupuncture (47\%), and herbs (39\%). $27 \%$ reported high adherence. Table 1 shows significant univariate associations with high adherence. In multivariate analysis adjusting for all variables in Table 1, only TCM use (OR 2.6, $p=0.027$ ) and higher MRCI (OR 1.1, $p=0.019)$ were associated with high adherence.

Table 1

\begin{tabular}{lccc}
\hline & $\begin{array}{c}\text { High adherence, } \\
\mathrm{n}=48\end{array}$ & $\begin{array}{c}\text { Med/Low adherence, } \\
\mathrm{n}=129\end{array}$ & $\mathrm{p}$-value \\
\hline Age, years (SD) & $63(11)$ & $52(17)$ & $<0.001$ \\
Employed, \% & 29 & 48 & 0.044 \\
$\geq 20$ years in US, \% & 58 & 39 & 0.02 \\
Age at immigration, years (SD) & $44(14)$ & $35(12)$ & $<0.001$ \\
RA, \% & 56 & 38 & 0.029 \\
MRCI, mean (SD) & $15(7)$ & $11(6)$ & 0.001 \\
TCM use, \% & 63 & 44 & 0.03 \\
PROMIS Sleep disturbance, T-score (SD) & $47(10)$ & $52(9)$ & 0.005 \\
PROMIS Anxiety, T-score (SD) & $46(11)$ & $49(10)$ & 0.04 \\
PROMIS Fatigue, T-score (SD) & $49(11)$ & $52(10)$ & 0.03 \\
\hline
\end{tabular}

*Lower score is better.

Conclusions: Among poorly integrated and low socioeconomic status CA rheumatology patients, TCM use was statistically significantly associated with high adherence to western medication, as was higher MRCI. TCM use does not appear to represent an alternate but rather complementary approach to disease management in these patients. Future studies should evaluate whether TCM use is associated with disease activity and outcomes over time.

\section{References:}

[1] Barton JL, et al. Racial and ethnic disparities in disease activity and function among persons with rheumatoid arthritis from university-affiliated clinics. Arthritis Care Res 2011;63:1238-1246. 